\title{
Analysis of Stress-Strain Curve of Polyethylene Films by Toda-Lattice Model
}

\author{
Koh-hei NitTa, Yuichi UCHIDA, and Akira TANAKA* \\ Department of Polymer Chemistry, Kyoto University, Kyoto 606, Japan
}

(Received November 5, 1990)

\begin{abstract}
Stress-strain curves of high density- and linear low density-polyethylenes and their blend films were analyzed by Toda-lattice model which has asymmetric interaction potential. Parameters in Toda-lattice model were experimentally determined from Young's modulus and Grüneisen parameter. Young's modulus was estimated from the initial slope of stress-strain curves, and Grüneisen parameter from the initial slope of ultrasonic velocity-strain curves. Using the parameters, we obtained the theoretical stress-strain curves at different temperatures for all the films. For all the films, the theoretical stress-strain curve was in good agreement with the experimental one except in the yielding region. Thus, nonlinear nature of polyethylene films can be ascribed mainly to the asymmetry of the interaction potential curve.

KEY WORDS Stress-Strain Curve / Toda-Lattice Model / Nonlinear Viscoelasticity / High-Density Polyethylene / Linear Low-Density Polyethylene / Blend / Grüneisen Parameter /
\end{abstract}

As is well known, polymeric solids, especially crystalline solids exhibit nonlinear viscoelasticity even at small strains, which is one of the indispensable properties of materials for practical use. In fact, the stress-strain relation of polyethylene films is nonlinear. It seems that the nonlinear relation cannot be ascribed to the viscoelastic nature, but to complicated morphology. Complicated morphology may cause various types of interactions such as inter-molecular and inter-aggregation interactions. Hence, the asymmetric interaction potential must be taken into account.

The Toda-lattice model is one of the models which have asymmetric interaction potential, and it has been introduced to theoretical studies on the nonlinear wave motion (soliton). ${ }^{1-3}$

In this paper, stress-strain curves for the films of high density- and linear low densitypolyethylenes and their blends were analyzed by the Toda-lattice model.

\footnotetext{
* To whom correspondence should be addressed.
}

\section{EXPERIMENTAL}

Various blend films of high density (linear; designated as HDPE) and linear low density (ethylene/butene-1 copolymer; designated as LLDPE) polyethylenes were used. Both the polyethylene samples were supplied from Mitsui Petrochem. Inc., Ltd. The number and weight average molecular weights were $1.3 \times$ $10^{4}, 7.0 \times 10^{4}$, respectively for HDPE and $1.4 \times 10^{4}$ and $1.0 \times 10^{5}$, respectively for LLDPE. The LLDPE polymer contains ethyl branches of about 3.7 per 100 backbone carbon atoms.

Pellets of the two polymers were blended at various proportions in a solution of xylene at $110^{\circ} \mathrm{C}$. The polymers were precipitated in methyl alcohol and then dried in a vacuum oven at room temperature. The following compositions were employed: A100/C0, A75/ $\mathrm{C} 25, \mathrm{~A} 50 / \mathrm{C} 50, \mathrm{~A} 25 / \mathrm{C} 75$, and $\mathrm{A} 0 / \mathrm{C} 100$. 
(Code A denotes HDPE, code C LLDPE, and numeral the percentage by weight of the materials.) They were melt pressed in a laboratory press for $10 \mathrm{~min}$ at $\mathrm{ca} .180^{\circ} \mathrm{C}$ and ca. $10 \mathrm{MPa}$, and quenched in ice-water bath $\left(0^{\circ} \mathrm{C}\right)$.

The density of the quenched films was determined by a floatation method. The binary medium prepared from various ratios of distilled water and ethyl alcohol was used. The densities of $\mathrm{A} 100 / \mathrm{C} 0, \mathrm{~A} 75 / \mathrm{C} 25, \mathrm{~A} 50 / \mathrm{C} 50$, $\mathrm{A} 25 / \mathrm{C} 75$, and $\mathrm{A} 0 / \mathrm{C} 100$ were $0.943,0.930$, $0.925,0.920$, and $0.912 \mathrm{~g} \mathrm{~cm}^{-3}$, giving the degrees of crystallinity of $60.7,51.7,48.7,44.8$, and $39.3 \%$, respectively.

The stress-strain and velocity-strain relations were simultaneously obtained using the apparatus previously reported. ${ }^{4}$ The elongation speed was ca. $9 \times 10^{-3} \mathrm{~m} \mathrm{~min}^{-1}$, and the length of film specimen was $c a .0 .025 \mathrm{~m}$. (Consequently, the strain rate was $c a .0 .6 \% \mathrm{~s}^{-1}$. The measurement were carried out at $30^{\circ}, 40^{\circ}$, and $50^{\circ} \mathrm{C}$.

\section{TODA-LATTICE MODEL}

The strain potential function of the Todalattice is of the form;

$$
\phi(\gamma)=a \gamma-(a / b)[1-\exp (-b \gamma)]
$$

where $a$ and $b$ are positive constants, $\gamma$ the strain, and $\phi(\gamma)$ the interaction energy ex- pressed as a function of strain. The two terms in the equation imply repulsive and attractive forces as a function of strain, respectively. The function is obviously anharmonic. When one keeps $a b=$ finite and takes the limit $b \rightarrow 0$, the equation becomes a quadratic function of $\gamma$ (harmonic case). On the other hand, when one takes $b \rightarrow \infty$, one gets the limit of hard sphere interaction. Thus, Toda-potential has wide applicability.

Figure 1 demonstrates an example of potential curves in the case of $a b=1$. As is shown in the figure, the larger the parameter $b$, the degree of distortion of the potential curve becomes greater.

Differentiating eq 1 by strain, one gets stress-strain relation;

$$
\sigma(\gamma)=a[1-\exp (-b \gamma)]
$$

As is obvious from eq 2 , the stress increases exponentially (or nonlinearly) with increasing strain.

If the stress is expanded in power series of $\gamma$,

$$
\begin{aligned}
\sigma(\gamma) & =a b \gamma-a b^{2} \gamma^{2} / 2+a b^{3} \gamma^{3} / 3 !-a b^{4} \gamma^{4} / 4 !+ \\
& =a b\left(\gamma-b^{2} \gamma^{2} / 2+b^{3} \gamma^{3} / 3 !-b^{4} \gamma^{4} / 4 !+\right)
\end{aligned}
$$

In eq 3 (or eq 4 ), all the elastic coefficients are expressed by the two parameters, $a$ and $b$, and the ratio of the higher order coefficient to the first order coefficient is expressed only by parameter $b$. This indicates that the anharmonicity or shape of potential curve is char-

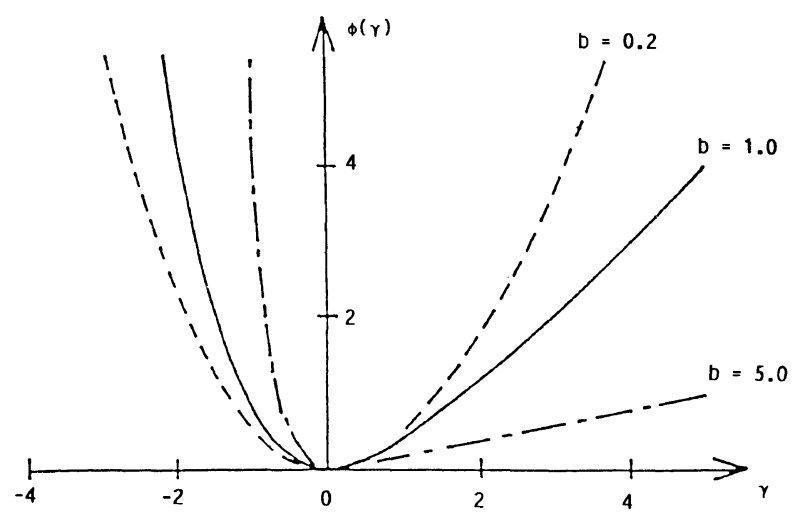

Figure 1. Example of the potential curve of Toda-Lattice Model in the case of $a b=1$. 
Table I. Young's modulus and Grüneisen parameter

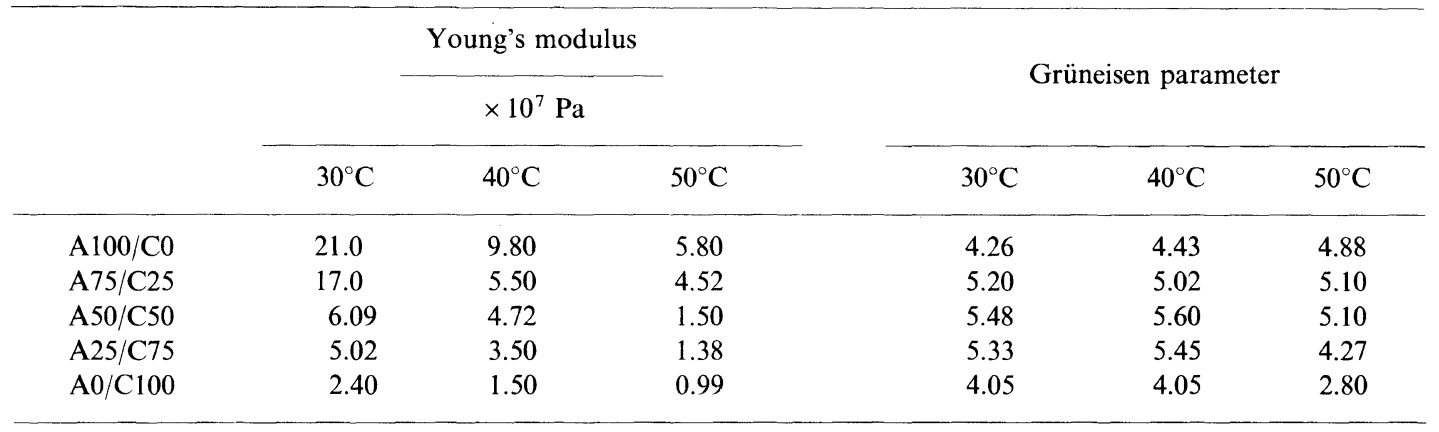

acterized by parameter $b$.

Again differentiating eq 2 by strain, one gets the modulus:

$$
E(\gamma)=a b \exp (-b \gamma)
$$

Young's modulus, $E_{0}$ (initial tangent modulus) can be obtained by putting $\gamma=0$ in eq 5:

$$
E_{0}=a b
$$

It should be noted that Young's modulus is expressed by the product of $a$ and $b$. Thus, the higher the Young's modulus, the higher is the nonlinearity of Toda-potential, when $a$ is constant.

Grüneisen parameter, $\gamma_{G}^{5,6}$ which represents the degree of anharmonicity of interaction potential $7^{7-10}$ is given by the following equation:

$$
\gamma_{\mathrm{G}}=-(1 / 2) \mathrm{d}(\ln E(\gamma)) / \mathrm{d} \gamma
$$

Substituting eq 5 into eq 7 , one can obtain the Grüneisen parameter of the Toda-potential:

$$
\gamma_{\mathrm{G}}=b / 2
$$

Thus, Grüneisen parameter is proportional to the parameter $b$. Consequently, the stressstrain relation can be formulated as a function of Young's modulus and Grüneisen parameter:

$$
\sigma(\gamma)=\left(E / 2 \gamma_{\mathrm{G}}\right)\left[1-\exp \left(-2 \gamma_{\mathrm{G}} \gamma\right)\right]
$$

Experimentally, Young's modulus was determined by the initial tangent slope of stressstrain curve and Grüneisen parameter by the initial slope of the ultrasonic velocity-strain curve, as described below.

\section{RESULTS AND DISCUSSION}

Stress-strain curves were obtained at $30^{\circ}$, $40^{\circ}$, and $50^{\circ} \mathrm{C}$ for HDPE, LLDPE, and their blend films. Young's moduli $\left(E_{0}\right)$ evaluated from the stress-strain curves are summarized in Table I.

Both stress and Young's modulus increased with increasing content of HDPE. For LLDPE-rich films, the stress monotonously increased with strain, and then it leveled off at higher strains. For HDPE-rich films (A100/C0 and $\mathrm{A} 75 / \mathrm{C} 25)$, on the other hand, the stress-strain curve exhibited a clear yield point around $20 \%$ of strain. With further increase in strain beyond the yield point, the specimen was nonuniformly deformed. No yield point was observed at higher temperatures; at $50^{\circ} \mathrm{C}$ for $\mathrm{A} 100 / \mathrm{C} 0$ and at $40^{\circ}$ and $50^{\circ} \mathrm{C}$ for $\mathrm{A} 75 / \mathrm{C} 25$ (see Figure 4).

Figure 2 shows the ultrasonic velocity-strain curves at $30^{\circ} \mathrm{C}$. The velocity decreased with increasing content of LLDPE. Moreover, the velocity decreased with increasing temperature, although the figure is not shown here. The decrease in the velocity can be ascribed to decrease in the modulus (or softening of the material).

The velocity increased linearly with increasing strain for all films. This increase can be due 


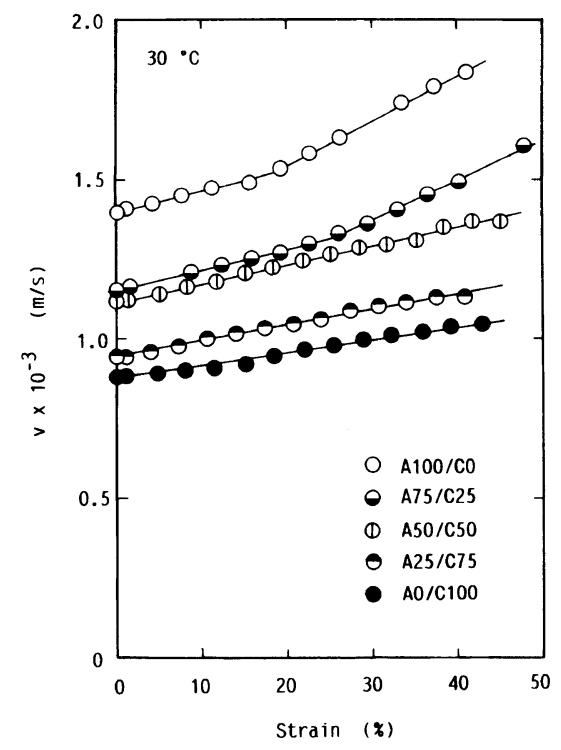

Figure 2. Ultrasonic velocity-strain curves of HDPE, LLDPE, and their blend films at $30^{\circ} \mathrm{C}$.

to the increase in the sonic modulus ${ }^{11,12}$ caused by the molecular orientation in the direction parallel to the stretching direction. ${ }^{13}$ For films in which yielding (or necking) phenomena appeared, however, the velocity-strain plot had a bending point around the yield point. The slope of the line became larger after the bending point. The larger slope (or bending) is considered due to underestimation of the strain in the necking region.

Grüneisen parameter $\gamma_{G}$ is defined as in the following equation ${ }^{14}$ :

$$
\gamma_{\mathrm{G}}=-\mathrm{d} \ln \omega / \mathrm{d} \varepsilon
$$

where $\omega$ is the intrinsic frequency of an oscillator and $\varepsilon$ the intermolecular strain. Since the change in velocity with strain corresponds to the change in the intrinsic frequency with strain, Grüneisen parameter can also be expressed as in the following equation ${ }^{15}$ :

$$
\gamma_{\mathrm{G}}=\mathrm{d} \ln v / \mathrm{d} \varepsilon
$$

Strain $\gamma$ defined in elongational tests is not always identical to the strain $\varepsilon$. Therefore, eq 11 can be rewritten as in the following equa-

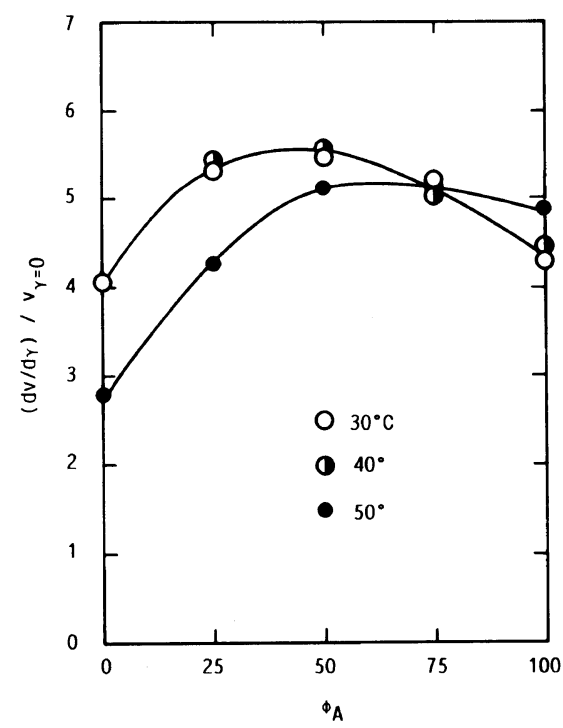

Figure 3. Variation in the Gruneisen parameter $\gamma_{G}$ with content of HDPE.

tion:

$$
\gamma_{\mathrm{G}}=(\mathrm{d} \gamma / \mathrm{d} \varepsilon)(\mathrm{d} \ln v / \mathrm{d} \gamma)
$$

where $(\mathrm{d} \ln v / \mathrm{d} \gamma)$ can be experimentally obtained as the initial slope of the velocity-strain curve. Wada et al. ${ }^{14,16}$ pointed out the following relation between $\gamma$ and $\varepsilon$.

$$
\gamma=(f / g)^{1 / 2} \varepsilon
$$

where $f$ is the intrachain force constant and $g$ the interchain force constant. The ultrasonic velocities in the direction parallel $\left(v_{\|}\right)$and perpendicular $\left(v_{\perp}\right)$ to the chain axis are proportional to $f$ and $g$, respectively. ${ }^{17,18}$ Accordingly, eq 12 can be rewritten as the following equation:

$$
\gamma_{\mathrm{G}}=\left(v_{\|} / v_{\perp}\right)(\mathrm{d} \ln v / \mathrm{d} \gamma)
$$

In the case of linear polyethylene, $\left(v_{\|} / v_{\perp}\right)$ becomes approximately $10 .^{18}$

The Grüneisen parameter thus obtained is plotted against the content of HDPE in Figure 3 , and it is also summarized in Table I. As is obvious from the figure and Table I, all the Grüneisen parameters are in the range from 3 to 6 , which are comparable to ones previously 

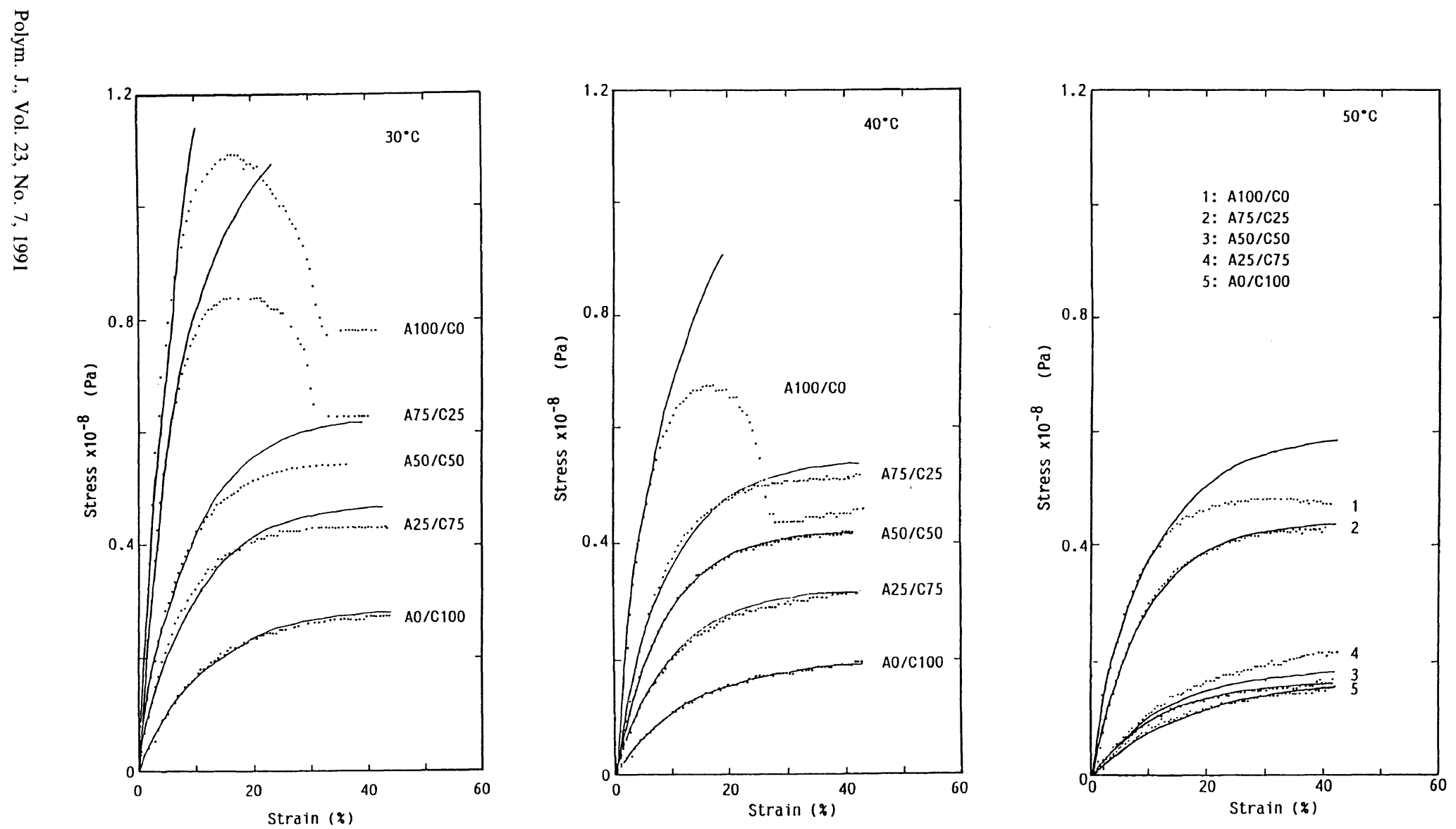

Figure 4. Comparison of experimental and theoretical stress-strain curves for HDPE, LLDPE, and their blend films at different temperatures. The solid line denotes the theoretical one, and the dotted line, the experimental one. 
obtained for polymeric materials. ${ }^{14,16,19-21} \mathrm{It}$ is noteworthy that the value of the blend polymers was higher than that of the pure polymers. This indicates that blending may enhance the anharmonicity of the interaction potential.

The theoretical stress-strain curves were obtained by putting the values $\left(E_{0}\right.$ and $\left.\gamma_{\mathrm{G}}\right)$ in Table I into eq 9. In Figure 4, the experimental and theoretical stress-strain curves are shown together. The solid line denotes the theoretical curve and the dotted line, the experimental one. As evident from the figure, the agreement between the theoretical and experimental curves was surprisingly good except in the strain region in which yielding phenomena appeared. This agreement suggests that the deformation mechanism can be satisfactorily represented by Toda-lattice model. The disagreement can be ascribed to the followings: (1) no correct strain can be obtained in the necking region, and (2) different deformation mechanisms such as plastic deformation (yielding), fibrous deformation, and so on may contribute to the velocity.

The shape of stress-strain curves for polymeric solid is strongly affected not only by temperature but also by strain-rate, as indicated by Ward. ${ }^{22}$ This indicates that polymeric solids deform as a typical viscoelastic body. Actually, stress-strain curves for polymeric solids have been analyzed by viscoelastic models. ${ }^{23-25}$

In a linear viscoelastic system with a single relaxation time $\tau$, the relaxation modulus can be expressed as in the following equation:

$$
E(t)=E_{0} \exp (-t / \tau)
$$

When one applies the above system to a special case in which the strain increases at a constant rate $(R=\mathrm{d} \gamma / \mathrm{d} t)$, the Boltzmann's superposition principle $^{26,27}$ gives the following stress-strain relation:

$$
\sigma(\gamma)=E_{0} R \tau[1-\exp (-\gamma / R \tau)]
$$

The relation can also be derived from Maxwell model when subjected to a constant rate of strain. It should be noticed that eq 11 is essentially identical to eq 9 . In other words, the stress-strain curves may be described also by the viscoelastic model.

We determined the relaxation time $(\tau)$ so as to give the best fit between the curve calculated by eq 11 and the experimental curve. The relaxation times thus obtained, however, were inconsistent with usual principle. The relaxation time sometimes increased with increasing temperature and with decreasing the degree of crystallinity (or the content of HDPE). Furthermore, the magnitudes were considerably small compared to those obtained from stress relaxation tests. ${ }^{28}$ This suggests that the nonlinearity of the stress-strain curve of polyethylene films is not ascribable to the viscoelastic effect, but to the asymmetric effect of interaction potential.

\section{REFERENCES}

1. M. Toda, J. Phys. Soc. Jpn., 22, 432 (1967).

2. M. Toda, J. Phys. Soc. Jpn., 23, 501 (1967).

3. N. J. Zabusky and M. D. Kruskal, Phys. Rev. Lett., 15, 240 (1965).

4. A. Tanaka, K. Nitta, and S. Onogi, Ploym. Eng. Sci., 29, 1124 (1989).

5. J. C. Slater, "Introduction to Chemical Physics," McGraw-Hill, New York, N. Y., 1966.

6. C. Kittel, "Introduction to Solid State Physics," John Wiley \& Sons, New York, N. Y., 1966.

7. O. Yamamoto, Polym. J., 2, 509 (1971).

8. T. Kojima, K. Koga, K. Imada, and M. Takayanagi, Polym. J., 7, 14 (1975).

9. E. L. Rodriguez and F. E. Filisko, J. Mater. Sci., 22, 1934, (1987).

10. R. M. Barron, T. H. K. Barron, P. M. Mummery, and M. Sharkey, Can. J. Chem., 66, 718 (1988).

11. M. Baccaredda and E. Butta, J. Polym. Sci., 22, 217 (1956).

12. P. D. Davidse, H. I. Waterman, and J. B. Westerdijik, J. Polym. Sci., 59, 389 (1962).

13. K. Adachi, G, Harrison, J. Lamb, A. M. North, and R. A. Pethrick, Polymer, 22, 1026 (1981).

14. Y. Wada, "Kobunshibussei to Bunshikouzo" (in Japanese), M. Nagasawa and Y. Yamashita, Ed., Kagakudoujin, 1973, p 113.

15. R. S. Bretslaff and R. R. Wool, J. Appl. Phys., 52, 
5964 (1981).

16. Y. Wada, A. Itani, T. Nishi, and S. Nagai, J. Polym. Sci., A-2, 7, 201 (1969).

17. R. E. Baker, J. Appl. Phys., 38, 4234 (1967).

18. W. W. Moseley, J. Appl. Polym. Sci., 3, 266 (1960).

19. M. G. Broadhurst and F. I. Mopsik, J. Chem. Phys., 52, 3634 (1970).

20. T. G. Gibon, J. Chem. Phys., 60, 1094 (1974).

21. D. S. Hughes, E. B. Blackenship, and R. L. Mims, J. Appl. Phys., 21, 294 (1950).

22. I. M. Ward, "Mechanical Properties of Solid Polymers, "John Wiley \& Sons, New York, N. Y.,
1971.

23. T. L. Smith, Trans. Soc. Rheol., 6, 61 (1962).

24. R. S. Stein, S. Onogi, K. Sasaguri, and D. A. Keedy, J. Appl. Phys., 34, 80 (1963).

25. S. Matsuoka, Polym. J., 17, 321 (1985).

26. H. Leaderman, "Elastic and Creep Properties of Filamentous Materials and Other High Polymers," The Textile Foundation, Washington D.C., 1943.

27. J. R. MacDonald and M. K. Brachmann, Rev. Mod. Phys., 28, 393 (1956).

28. S. Onogi, A. Tanaka, Y. Ishikawa, and T. Igarashi, Polym. J., 7, 467 (1975). 